\title{
Transverse compression of an intense ion beam propagating through an alternating-gradient quadrupole lattice
}

\author{
Mikhail Dorf, Ronald C. Davidson, and Edward A. Startsev \\ Plasma Physics Laboratory, Princeton University, Princeton, New Jersey 08543, USA
}

(Received 28 November 2005; published 15 March 2006)

\begin{abstract}
The transverse compression and dynamics of an intense beam propagating through an alternatinggradient quadrupole lattice, plays an important role in many accelerator physics applications. Typically, the compression can be achieved by means of increasing the focusing strength of the lattice along the beam propagation direction. However, beam propagation through the lattice transition region inevitably leads to a certain level of beam mismatch and halo formation. In this work we present a detailed analysis of these phenomena using the envelope equations in the smooth-focusing approximation, which describe the average effects of an alternating-gradient lattice, and full particle-in-cell numerical simulations using the WARP code, taking into account the effects of the alternating-gradient quadrupole field. Simulations are presented for both space-charge-dominated beams, and beams with a moderate space-charge strength.

DOI: 10.1103/PhysRevSTAB.9.034202

PACS numbers: 41.85.Ja, 52.59.Sa, 52.59.Tb
\end{abstract}

\section{INTRODUCTION}

Alternating-gradient accelerators and transport systems have a wide range of applications ranging from basic scientific research to industrial applications [1-3]. Of considerable practical importance for heavy ion beam applications to high energy density physics and fusion is the axial compression and transverse focusing of the (initially long) charge bunch to a small spot size at the target location. One of the modern approaches to the compression process is to use dense background plasma which charge neutralizes the ion charge bunch, and hence facilitates compression of the bunch against strong space-charge forces [4-7]. On the other hand, the transverse focusing can also be achieved by means of increasing the focusing strength of the alternating-gradient lattice along the beam propagation direction. Although lattice compression significantly facilitates the technical realization of the process, uncompensated, high-intensity charge bunch propagation through the lattice transition region inevitably leads to a certain level of beam mismatch and emittance growth. Furthermore, it is well known that a beam mismatch can produce halo particles [8-10] that have much higher transverse energy than the core particles and may cause a degradation of beam quality.

It is evident that the beam mismatch will decrease as the length of the transition region is increased, assuming that the lattice amplitude is constant outside the transition region. Hence, it is a matter of considerable practical interest to determine how smooth (adiabatic) the lattice transition should be to assure that matching is maintained during the compression. In Sec. II, a detailed investigation of this problem is performed for a long, coasting beam using the envelope equations and full particle-in-cell numerical simulations with the WARP code [11] in the smooth-focusing approximation, which describe the average effects of a periodic lattice. In Sec. III the effects of the alternating-gradient quadrupole field are taken into account. It is found that even a strong mismatch, produced during the compression process, can be consistent with moderate emittance growth. Nonetheless a certain fraction of the beam particles experience resonant interaction with the mismatch oscillations and populate the halo region. In Sec. IV, the details of halo formation are investigated selfconsistently for the case of an alternating-gradient quadrupole lattice, and the results are shown to agree well with the particle-core model developed by Ikegami [12].

In the analysis performed in this paper, a stable transport regime is assumed during the compression. Hence, the characteristic features of the beam compression that are described in Secs. II and III will be similar for a wide range of intense beams with similar dimensionless parameters satisfying the stable transport condition $\sigma_{\text {vac }}^{2}-\sigma^{2}<$ $\left(120^{0}\right)^{2} / 2[13,14]$. Here, $\sigma$ and $\sigma_{\text {vac }}[2,15]$ (both measured in degrees per lattice period) denote the phase advance of particles oscillating in a periodic focusing lattice in the presence and absence of beam space charge, respectively. The undepressed phase advance $\sigma_{\text {vac }}$ measures the strength of the applied focusing force of the lattice and is relatively insensitive to the details of the lattice waveform. The ratio $\sigma / \sigma_{\text {vac }}$ is a normalized measure of the space-charge strength with $\sigma / \sigma_{\text {vac }} \rightarrow 1$ corresponding to an emittancedominated beam with negligible space-charge force, and $\sigma / \sigma_{\text {vac }} \rightarrow 0$ corresponding to a space-charge-dominated beam with negligible emittance.

The transverse compression of a long axially-stationary charge bunch is currently being investigated in the Paul Trap Simulator Experiment (PTSX) [16] that simulates the nonlinear transverse dynamics of intense beam propagation over large distances through an alternating-gradient transport lattice. Since we study only transverse beam dynamics in the present analysis, it is convenient to perform the analysis in the axial rest frame of the charge bunch. In this frame axial ion velocity is equal to zero, 
$V_{b}=0$, and the relativistic mass factor is equal to unity $\left(\gamma_{b}=1\right)$. It should be noted that the axial rest frame of the charge bunch, described above, is the laboratory frame for the experiments carried out on PTSX.

\section{SMOOTH-FOCUSING APPROXIMATION}

In this section we make use of the so-called smoothfocusing approximation [1-3] to study the nonlinear response of long charge bunches to alternating-gradient waveforms with time-varying amplitude. This model is used to describe the average effects of a quadrupole focusing field. Within this approximation, the external focusing potential has the form

$$
\varphi_{\mathrm{foc}}(\vec{r}, t)=\frac{1}{2} \frac{m_{b}}{e_{b}} \omega_{q}^{2}(t) r^{2},
$$

where $\omega_{q}(t)$ is the smooth-focusing frequency, $r$ is the radial distance from the beam axis, and $e_{b}$ and $m_{b}$ are the particle mass and charge, respectively. For present purposes, it is convenient to introduce the normalized intensity parameter $s \equiv \omega_{p}^{2} / 2 \omega_{q}^{2}$, where $\omega_{p}=$ $\left(4 \pi n_{0} e_{b}^{2} / m_{b}\right)^{1 / 2}$ is the plasma frequency, and $n_{0} \equiv n(r=$ 0 ) is the on-axis number density.

The initial quasistationary distribution, which is used in the simulations later in this section, is assumed to correspond to a thermal equilibrium distribution [1], and the corresponding density profile $n(r)$ is given by

$$
n(r)=n_{0} \exp \left[-\frac{e_{b} \varphi_{\mathrm{foc}}(r, 0)+e_{b} \varphi^{s}(r)}{T}\right] .
$$

Here, $T=$ const is the transverse temperature, and the space-charge potential $\varphi^{s}(r)$ is determined selfconsistently from Poisson's equation $\nabla^{2} \varphi^{s}(r)=$ $-4 \pi e_{b} n(r)$. Except for space-charge-dominated beams $(s \rightarrow 1)$, numerical solutions of Poisson's equation show that the radial density profile is bell shaped, and is nearly Gaussian even for moderate values of $s$. Regardless of the detailed shape of the density profile, the mean-square radius $R_{b}^{2}$ of the charge bunch is determined from the global radial force balance constraint [1]

$$
m_{b} \omega_{q}^{2} R_{b}^{2}=2 T+N_{b} e_{b}^{2},
$$

where $N_{b}=2 \pi \int_{0}^{r_{w}} d r r n(r)$ is the line density, $r_{w}$ is the wall radius, and $R_{b}^{2}=2 \pi N_{b}^{-1} \int_{0}^{r_{w}} d r r^{3} n(r)$ is the meansquare beam radius.

\section{A. Rate equation for rms beam radius}

From the fully nonlinear Vlasov-Maxwell equations describing a long charge bunch when the external focusing force has cylindrical symmetry [Eq. (1)], one can derive the following equation that describes the evolution of the rms radius of the charge bunch [1]

$$
\frac{d^{2}}{d t^{2}} R_{b}+\left(\omega_{q}^{2}(t)-\frac{K_{b}}{2 R_{b}^{2}}\right) R_{b}=\frac{\varepsilon^{2}(t)}{4 R_{b}^{3}} .
$$

Here, $K_{b}=2 N_{b} e_{b}^{2} / m_{b}$ is the effective self-field perveance, and $\varepsilon=\left(4 R_{b}^{2}\left[\left\langle\dot{x}^{2}+\dot{y}^{2}\right\rangle-\dot{R}_{b}^{2}\right]\right)^{1 / 2}$ is the unnormalized transverse emittance defined in the beam frame. The super$\operatorname{dot}(\cdot)$ denotes time derivative and $\langle\ldots\rangle$ denotes the statistical average over the particle distribution function $f_{b}(x, y, \dot{x}, \dot{y}, t)$ in the transverse phase space $(x, y, \dot{x}, \dot{y})$. Although the emittance will vary due to nonuniformities in charge density, for present purposes we assume that $\varepsilon(t)$ is approximately constant if the focusing frequency $\omega_{q}(t)$ changes adiabatically. More detailed studies of the emittance behavior are presented in Sec. II B. Assuming that $\varepsilon(t)=$ const we can use Eq. (4) to analyze the evolution of the rms beam radius during the compression process. To describe the compression, we adopt a simple model in which $\omega_{q}(t)$ varies according to [17]

$$
\omega_{q}(t)=\omega_{q i}-\left(\omega_{q f}-\omega_{q i}\right)\left[\exp \left(\frac{\tau_{1 / 2}-t}{\tau_{q}}\right)+1\right]^{-1},
$$

where $2 \tau_{1 / 2}$ is the characteristic transition time, and $\tau_{q}$ is the characteristic time scale for variation of $\omega_{q}(t)$ from the constant value $\omega_{q i}$ to the constant value $\omega_{q f}$.

Here, we consider long charge bunches, which are initially matched. This readily gives for the smooth-focusing model that $\left[\dot{R}_{b}\right]_{t=0}=0$ and $\left[\ddot{R}_{b}\right]_{t=0}=0$. Using the simplified Eq. (4), we now estimate the transition time $2 \tau_{1 / 2}$ that is consistent with adiabatic compression. For a quantitative description of the adiabaticity of the compression process we introduce the beam mismatch parameter $\eta=$ $\Delta R / R_{0}$, where $\Delta R$ is the amplitude of the mismatch oscillations, and $R_{0}(t)$ is the quasiequilibrium radius, which is determined from the instantaneous value of $\omega_{q}(t)$. Here, note that $\Delta R+R_{0}=R_{b}$. Assuming that $\eta \ll 1$ during the adiabatic compression, we linearize Eq. (4) around the quasiequilibrium radius $R_{0}(t)$, which gives

$$
\Delta \ddot{R}+\bar{\omega}^{2}(t) \Delta R=-\ddot{R}_{0},
$$

where $\bar{\omega}=\left(K_{b} / R_{0}^{2}+\varepsilon^{2} / R_{0}^{4}\right)^{1 / 2}$ is the frequency of smallamplitude linear oscillations. It is evident from Eq. (6) that for adiabatic compression the inverse transition time $\left(2 \tau_{1 / 2}\right)^{-1}$ has to be much smaller than $\bar{\omega}$. Note, that the frequency $\bar{\omega}$ depends on $R_{0}$, and hence the inverse transition time needs to be much smaller than its minimum value, i.e., $\left(2 \tau_{1 / 2}\right)^{-1} \ll \bar{\omega}\left(R_{0 i}\right)$, where $R_{0 i}$ is the initial quasiequilibrium beam radius. For more detailed studies of the adiabaticity of the process we make use of the Van Der Pol method and introduce the following variables: $z=$ $\Delta \dot{R}+i \bar{\omega}(t) \Delta R$ and $a=(1 / \sqrt{\bar{\omega}}) z \exp \left(-i \int_{0}^{t} \bar{\omega} d t\right)$. Note that $|z|^{2} / 2=\left(\Delta \dot{R}^{2}+\bar{\omega}^{2} \Delta R^{2}\right) / 2=E$ is the energy of the oscillator, and $|a|^{2}=|z|^{2} / \bar{\omega}$ is a well-known adiabatic invariant for the pendulum Eq. (6). The physical interpretation of this adiabatic invariant corresponds to the area of 
the ellipse in the phase space $(\Delta R, \Delta \dot{R})$. After some straightforward algebra, we obtain the following equation for the evolution of the adiabatic invariant:

$$
\dot{a}=-\frac{\dot{\bar{\omega}}}{\bar{\omega}} a^{*} \exp \left(-2 i \int_{o}^{t} \bar{\omega} d t\right)-\frac{\ddot{R}_{0}}{\sqrt{\bar{\omega}}} \exp \left(-i \int_{0}^{t} \bar{\omega} d t\right),
$$

where star $\left(^{*}\right)$ denotes complex conjugate. Recall that before compression the beam is matched, with $a(0)=0$. Furthermore, the transition time should be large enough to

$\mathrm{t} / \tau_{\mathrm{q}}$ (adiabatic compression)
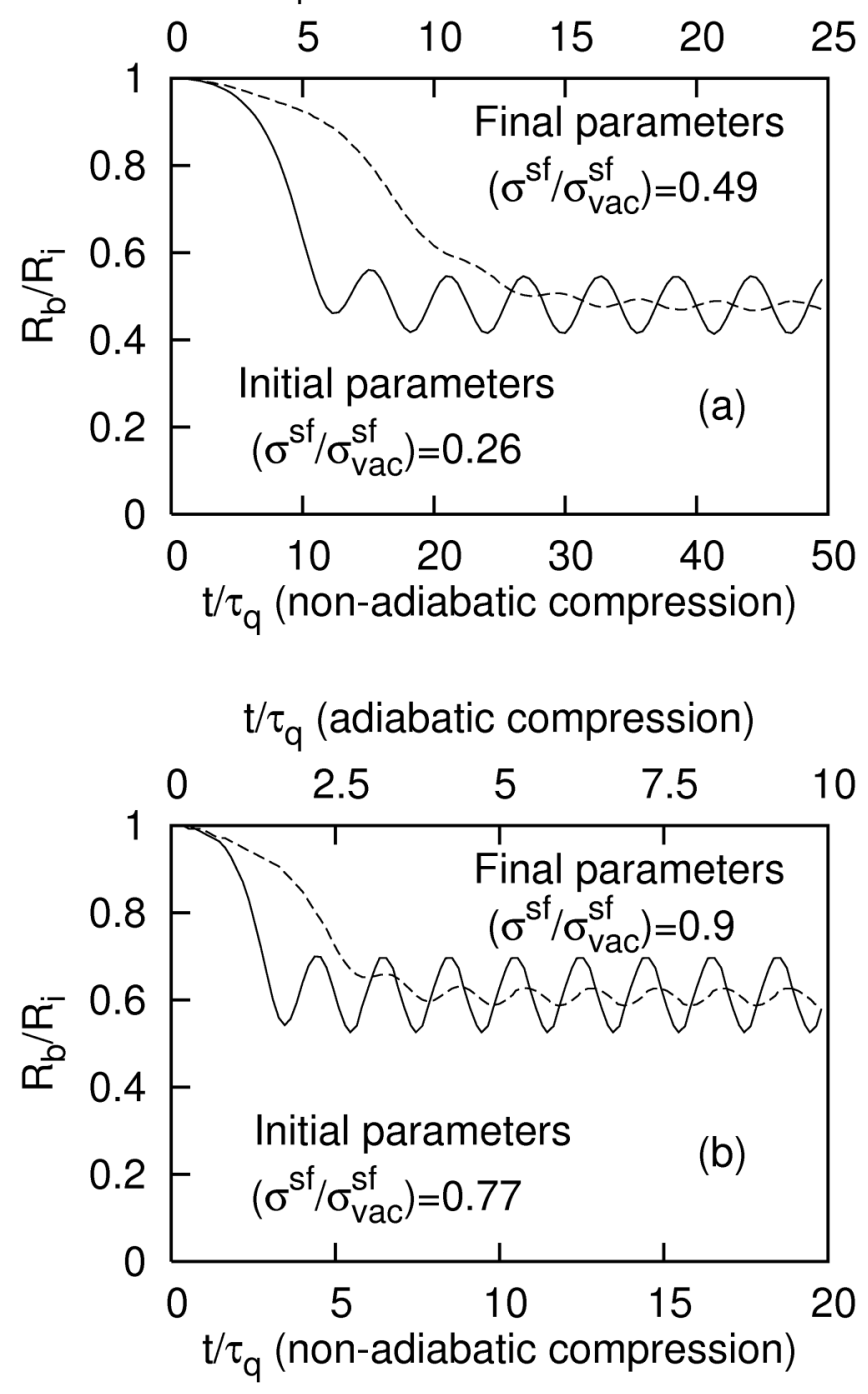

FIG. 1. Numerical solutions to envelope Eq. (4) for $\varepsilon=$ const. Plots of normalized rms beam radius $R_{b} / R_{i}$ versus $t / \tau_{q}$. The cases shown correspond to: (a) Space-charge-dominated beam with $s=0.9999$; broken line: adiabatic compression with $\tau_{q} \bar{\omega}\left(R_{0 i}\right)=1.74$; solid line: nonadiabatic compression with $\tau_{q} \bar{\omega}\left(R_{0 i}\right)=0.87$. (b) Moderate space-charge strength with $s=$ 0.7 ; broken line: adiabatic compression with $\tau_{q} \bar{\omega}\left(R_{0 i}\right)=1.28$; solid line: nonadiabatic compression with $\tau_{q} \bar{\omega}\left(R_{0 i}\right)=0.64$. assure $\eta \ll 1$. Hence we can neglect the first term on the right-hand side of Eq. (7). As a result, we obtain

$$
a(t) \cong-\int_{0}^{t} \frac{\ddot{R}_{0}}{\sqrt{\bar{\omega}}} \exp \left(-i \int_{0}^{t^{\prime}} \bar{\omega} d t^{\prime}\right) d t
$$

It is evident from Eq. (8) that the inverse transition time $\left(2 \tau_{1 / 2}\right)^{-1}$ must be much smaller than the frequency of linear oscillations $\bar{\omega}$ to assure that matching is maintained during compression. In this case $|a|^{2} \propto \exp \left(-\langle\bar{\omega}\rangle \tau_{q}\right)$, where $\langle\bar{\omega}\rangle$ is a certain value $\bar{\omega}$ of between $\bar{\omega}\left(R_{0 i}\right)$ and $\bar{\omega}\left(R_{0 f}\right)$, and $R_{0 i}, R_{0 f}$ are the initial and final quasiequilibrium beam radii, respectively.

Illustrative numerical solutions to Eq. (4) are presented in Fig. 1. To model a warm beam with moderate spacecharge strength, and a space-charge-dominated beam, we consider the cases $s=0.7$ and $s=0.9999$, respectively. For the compression, we take $\tau_{1 / 2} / \tau_{q}=5$ and $\omega_{q f} / \omega_{q i}=$ 2.3 in Eq. (5). Figure 1 shows that a relatively fast compression, $\tau_{q} \bar{\omega}\left(R_{0 i}\right)=0.87$ for $s=0.9999$, and $\tau_{q} \bar{\omega}\left(R_{0 i}\right)=0.64$ for $s=0.7$, leads to a significant mismatch in the final state, whereas a more adiabatic compression, $\quad \tau_{q} \bar{\omega}\left(R_{0 i}\right)=1.74$ for $s=0.9999$, and $\tau_{q} \bar{\omega}\left(R_{0 i}\right)=1.28$ for $s=0.7$, provides a nearly matched beam envelope in the final stage. Figure 2 shows how the characteristic time scale for variation of $\omega_{q}(t)$ depends on the ratio of the final to initial beam radius, for adiabatic compression, and for several values of $s$. To estimate the transition time, we use the condition that the final mismatch parameter, $\eta_{f}=\Delta R / R_{0 f}$, is equal to or less than $2 \%$. To describe the change of the intensity parameter during the compression process, the ratio of the phase advances $\sigma^{s f} / \sigma_{\text {vac }}^{s f}=\left[1+\left(K_{b} / 2 \varepsilon \omega_{q}\right)^{2}\right]^{1 / 2}-$ $\left(K_{b} / 2 \varepsilon \omega_{q}\right)$, calculated in the smooth-focusing approxima-

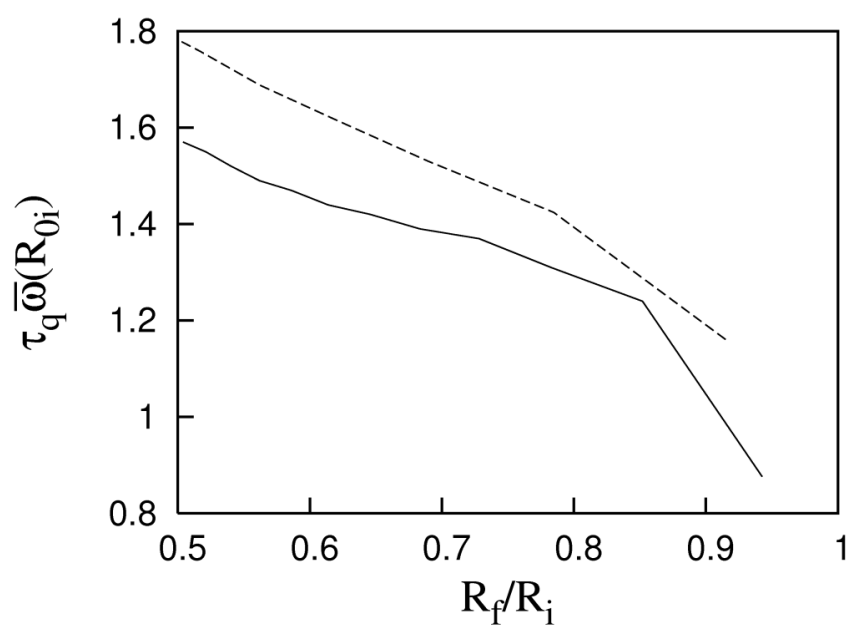

FIG. 2. Plot of $\tau_{q} \bar{\omega}\left(R_{0 i}\right)$ versus $R_{f} / R_{i}$ for adiabatic compression with $\eta_{f}=2 \%$. The two cases correspond to normalized intensity $s=0.9999$ (broken curve) and $s=0.7$ (solid curve). 


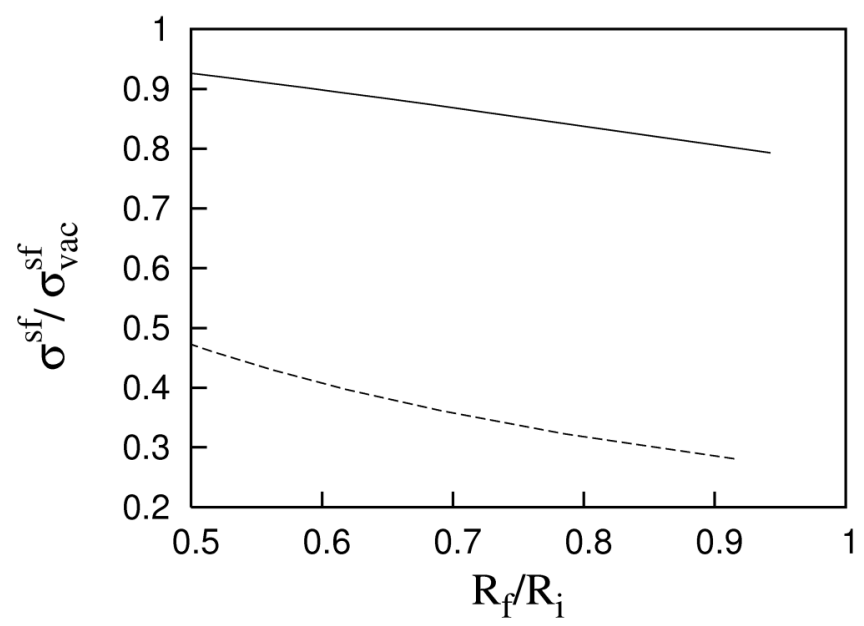

FIG. 3. Plots of $\sigma / \sigma_{\text {vac }}$ versus $R_{f} / R_{i}$ for adiabatic compression with $\eta_{f}=2 \%$. The two cases correspond to normalized intensity $s=0.9999$ (broken curve) and $s=0.7$ (solid curve).

tion [1] for different values of the final beam radius, is plotted in Fig. 3.

It should be noted from Fig. 3 that the relative spacecharge strength as measured by $\sigma^{s f} / \sigma_{\text {vac }}^{s f}$ decreases during the adiabatic compression process. This result can be explained by recalling that $\varepsilon(t) \approx$ const is assumed during the adiabatic process. Since $\left\langle\dot{x}^{2}+\dot{y}^{2}\right\rangle \gg \dot{R}_{b}^{2}$ for slow (adiabatic) compression, we obtain the following relation between the beam radius and effective transverse temperature, $R_{b} T^{1 / 2} \approx$ const. Therefore, decreasing the beam radius results in an increase in the effective transverse beam temperature, and hence a decrease in the relative spacecharge strength, which can also be measured by the parameter $\delta=N_{b} e_{b}^{2} / 2 T$ in Eq. (3) [1].

\section{B. Numerical simulations of beam compression with the WARP code}

The analysis of beam compression presented in Sec. II A was made under the assumption that the transverse emittance remains approximately constant during the adiabatic compression process. To elucidate the details of the emittance behavior, the fully nonlinear Vlasov-Maxwell equations should be solved. In this section, we employ a two-dimensional transverse slice model using the WARP electrostatic particle-in-cell (PIC) code [11] for this purpose. Results of the numerical simulations for the illustrative parameters used in Sec. II A are shown in Fig. 4. Evidently, there is no significant emittance change during the adiabatic compression process. For a space-chargedominated beam with $s=0.9999$, the emittance decreases by $4 \%$ from its initial value, and for moderate space-charge strength with $s=0.7$, the emittance variations are less than $1 \%$. Such a small emittance change during the adiabatic
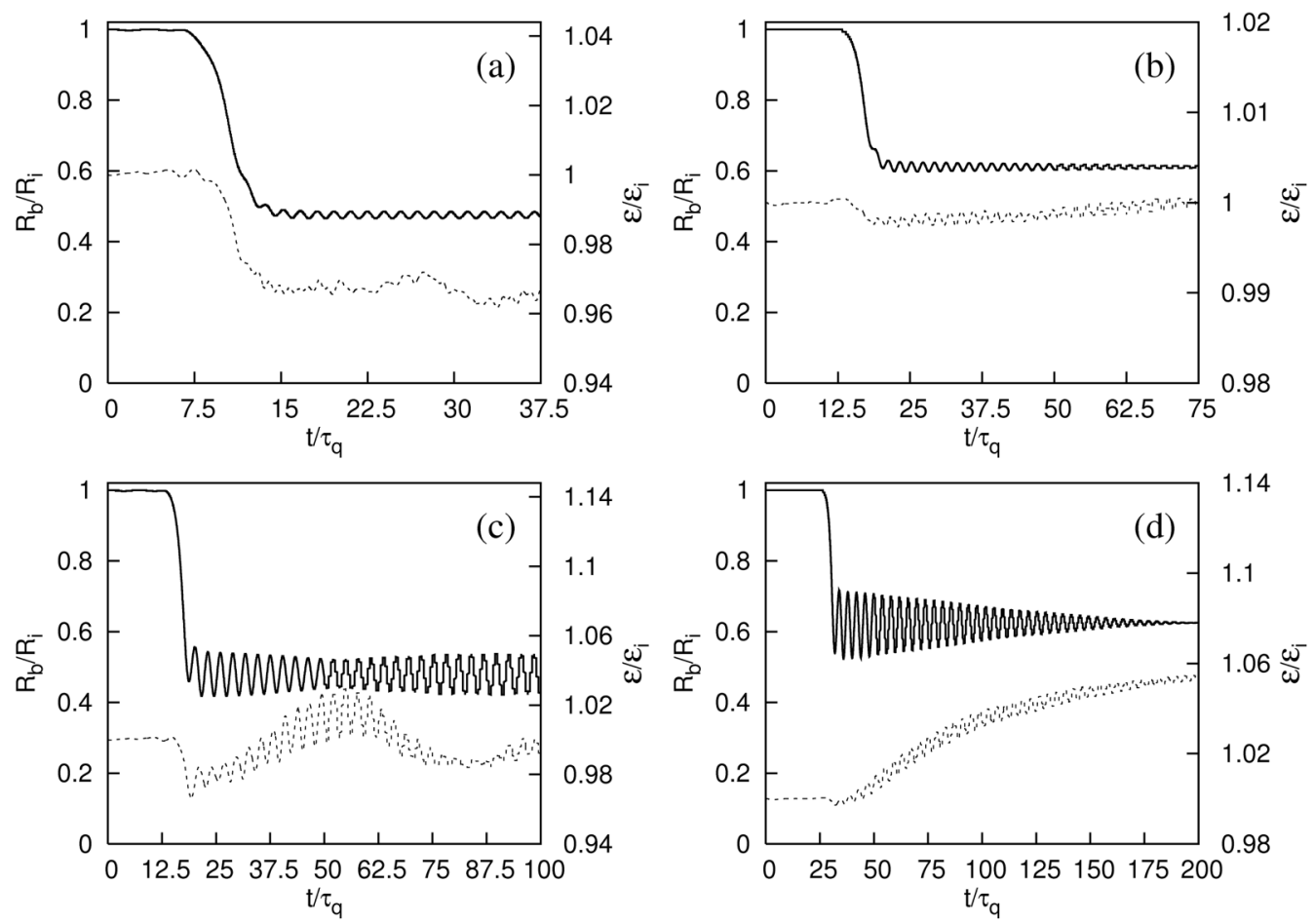

FIG. 4. Evolution of the normalized beam radius $R_{b} / R_{i}$ (solid line) and normalized transverse emittance $\varepsilon / \varepsilon_{i}$ (broken line) during the compression process. (a),(b) Correspond to an adiabatic compression for $s=0.9999, \tau_{q} \bar{\omega}\left(R_{0 i}\right)=1.74$, and for $s=0.7$, $\tau_{q} \bar{\omega}\left(R_{0 i}\right)=1.28$, respectively. (c),(d) Correspond to a nonadiabatic compression for $s=0.9999, \tau_{q} \bar{\omega}\left(R_{0 i}\right)=0.87$, and for $s=$ $0.7, \tau_{q} \bar{\omega}\left(R_{0 i}\right)=0.64$, respectively. Results are obtained using the WARP code for a smooth-focusing field. 
compression process validates the assumptions made in Sec. II A. For the case of nonadiabatic compression, when the transition time is small compared to the inverse frequency of beam radius oscillations, $\bar{\omega}^{-1}$, the emittance variations are nearly $6 \%$ in both cases.

Despite such a moderate emittance change, an important qualitative difference is evident for the time evolution of the beam radius, when comparing results from the PIC code simulations and from the constant-emittance model. To describe this phenomenon, it is convenient to introduce two stages of the compression process. The transition stage takes place during the transition phase of the smoothfocusing frequency, $\omega_{q}(t)$, i.e., $0<t<2 \tau_{1 / 2}$, and the relaxation stage for $t>2 \tau_{1 / 2}$ represents the mismatched beam behavior in the final focusing field with constant smooth-focusing frequency $\omega_{q f}$. The largest difference in behavior is observed in the relaxation stage, during the nonadiabatic process when the beam is strongly mismatched after the transition. Figure 1 shows that the constant-emittance model exhibits oscillations in beam radius with a constant amplitude, whereas the fully nonlinear Vlasov-Maxwell description gives a slight damping of the oscillations for a space-charge-dominated beam with $s=0.9999$ [Fig. 4(c)], and an almost complete mixing of the oscillations for the moderate space-charge strength with $s=0.7$ [Fig. 4(d)].

A plausible description of the damping mechanism of the mismatched oscillations is the following. Nonuniformities in the density profile produce nonlinear self-fields. Therefore, particles move with energydependent betatron frequency and affect the oscillations of moments of the distribution function due to phasemixing. References $[18,19]$ give a detailed explanation of these phenomena by means of Landau damping. In Ref. [18] the particles are considered as an ensemble of betatron oscillators coupled to the collective mismatch oscillations (mismatch mode). The damping of the mismatch mode occurs due to the energy transfer from collective oscillations to the oscillators (beam particles) which are close to resonance with the mismatch mode. The relaxation time is determined by the phase mixing of the trapped particles (resonant betatron oscillators). In the same work [18] it is shown that for the case of a spacecharge-dominated beam most of the betatron oscillators are far from resonance, providing a slight damping of the collective oscillations. As the beam space-charge intensity decreases, the mismatch frequency approaches the frequency distribution of the betatron oscillators, providing an increased mixing of the collective oscillations. This coincides well with the results obtained in the present paper. Here, we also emphasize that the present simulations show (Fig. 5) that a large mismatch for a spacecharge-dominated beam tends to relax to a state with a nonuniform density profile, and to a distribution function which is periodic in time. This state corresponds qualita-
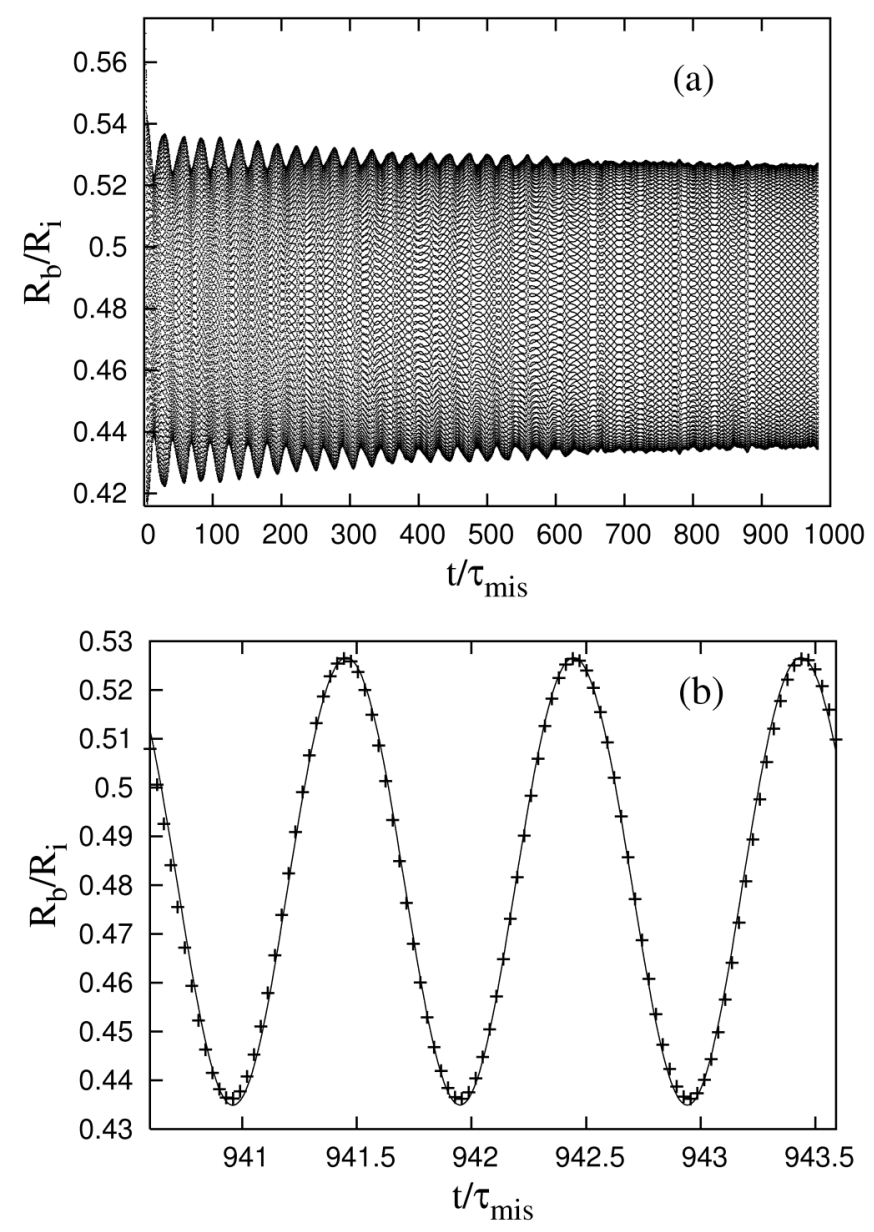

FIG. 5. Relaxation of the large mismatch in a space-chargedominated beam with $s=0.9999$ during the compression process using the smooth-focusing approximation. (a) Plot of $R_{b} / R_{i}$ verses normalized time $t / \tau_{\text {mis }}$, where $\tau_{\text {mis }}$ is the period of the mismatched oscillations in the final state. (b) Time dependence of $R_{b} / R_{i}$ in the final state (solid line) can be fitted with a cosine function (crosses) with high accuracy. The ratio of the first and second harmonic amplitudes in the spectrum of the $R_{b}(t)$ dependence (obtained by applying fast-Fourier-transform techniques) is equal to $2.5 \times 10^{4}$. Results are obtained using the WARP code.

tively to the nonlinear saturation of Landau damping and has a significant number of trapped particles (see Sec. IV for details). Future studies of this state may provide important insights for the construction of "equilibrium" states for intense beam propagation in a periodic lattice.

The relaxation process described above transfers energy from the collective oscillations to the transverse motion of the resonant particles, thereby increasing the transverse phase-space area (emittance growth). Figure 4(d) (for the beam radius) indicates that the phase-mixing time is about 30 times larger than the transition time. Hence, it is expected that there will be negligible emittance variations during the transition stage even during nonadiabatic compression. Indeed, Fig. 4(d) (for the emittance) shows that the emittance decrease during the initial transition of the 
smooth-focusing frequency is less than $0.5 \%$, whereas the overall emittance growth is $6 \%$. A theoretical model providing the estimate of the emittance growth due to the mismatch relaxation can be found in [2]. The detailed behavior of the emittance validates the use of Eq. (4) with the $\varepsilon=$ const assumption to model the transition stage, even for nonadiabatic compression. Comparing Figs. 1 and 4 indicates that the constant-emittance model and the PIC simulations give approximately the same initial amplitude of the mismatched oscillations.

Another interesting feature of the compression process is the emittance decrease during the initial transition stage. Examining Fig. 4 shows that the emittance decrease depends weakly on the transition time, and it is much larger for a space-charge-dominated beam than for a moderate intensity beam. To explain this phenomenon, we make use of the rate equation for the transverse emittance [1,2],

$$
\frac{1}{8} \frac{d}{d t} \varepsilon^{2}=-R_{b}^{2} \frac{d}{d t}\left(E_{F}-E_{K V}\right) .
$$

Here, $E_{F}=\left(2 K_{b}\right)^{-1} \int_{0}^{r_{w}} d r r\left|\nabla_{\perp} \psi\right|^{2}$ is the normalized selffield energy, $\psi$ solves $\nabla^{2} \psi=-\left(2 \pi K_{b} / N_{b}\right) \iint d \dot{x} d \dot{y} f_{b}$, and $E_{K V}=(1 / 2) K_{b}\left(1 / 4+\ln \left[r_{w} /\left(2^{1 / 2} R_{b}\right)\right]\right)$ is the selffield energy of the equivalent cold $(T=0)$ beam, having the same rms radius $R_{b}$ and line density $N_{b}$. It can be shown that the self-field energy of the thermal equilibrium beam [Eq. (2)] with fixed rms radius and line density decreases with decreasing temperature and reaches its minimum value for a cold distribution $(T=0)$ with the flattop density profile. During the transition stage, the effective beam temperature increases [see Sec. II B for details], thereby increasing the difference between $E_{F}$ and $E_{K V}$ and leading to a decrease in the emittance.

\section{ALTERNATING-GRADIENT QUADRUPOLE FIELD}

In this section, we present numerical studies using the WARP code [11] describing the beam response to an alternating-gradient quadrupole focusing field with timevarying amplitude. One of the major challenges in performing such simulations is to load the particles in such a way as to provide an initial "quasiequilibrium" of the beam. Within the smooth-focusing model used in Sec. II, the oscillating strength of the lattice was replaced with a constant focusing force. This simplification makes the external focusing potential time independent. Therefore any distribution function $f_{b}(x, y, \dot{x}, \dot{y}, t)$ that depends only on the particle's Hamiltonian, $f_{b}=F(H)$, provides a steady-state beam equilibrium with $\partial f_{b} / \partial t \equiv 0$. For the numerical simulations performed in Sec. II B we considered a thermal equilibrium beam with $F=\left(n_{0} / 2 \pi m_{b} T\right) \times$ $\exp [-H / T]$, where $H=\left(m_{b} / 2\right)\left(\dot{x}^{2}+\dot{y}^{2}\right)+e_{b} \varphi_{\mathrm{foc}}(r)+$ $e_{b} \varphi^{s}(r)$. In this section, the external focusing potential is periodic in time, and the condition $\partial f_{b} / \partial t \equiv 0$ is not satisfied. Therefore, we define a beam "equilibrium" as a state in which the beam is "matched", i.e., the distribution function is periodic with the lattice period $\tau_{L}$, $f_{b}(x, y, \dot{x}, \dot{y}, t)=f_{b}\left(x, y, \dot{x}, \dot{y}, t+\tau_{L}\right)$. The only known distribution function that provides an exact beam equilibrium in an alternating-gradient quadrupole field is the Kapchinskij-Vladimirskij (KV) distribution [1], but this distribution is unstable for sufficiently high beam intensities.

In the present simulations, the following scheme is used to load the initial distribution function. First, for a specified intensity parameter $s$, effective temperature $T$, and on-axis number density $n_{0}$, we apply the smooth-focusing model to construct the initial equilibrium. Then, using the corresponding values for the emittance $\varepsilon$ and perveance $K_{b}$, we determine the matched solutions of the envelope Eqs. (10) for a periodic focusing quadruple field [1],

$$
\begin{aligned}
& \ddot{a}+k_{q}(t) a-2 K_{b} /(a+b)=\varepsilon_{x}^{2} / a^{3}, \\
& \ddot{b}-k_{q}(t) b-2 K_{b} /(a+b)=\varepsilon_{y}^{2} / b^{3} .
\end{aligned}
$$

Here $a(t)$ and $b(t)$ are the half-widths of the transverse beam dimensions in the $x$ and $y$ directions, respectively, $\varepsilon_{x}=\varepsilon_{y}=\varepsilon$ are the transverse emittances, and $k_{q}(t)$ is the alternating-gradient lattice function [1,2]. Matched solutions to Eq. (10) correspond to solutions that satisfy $a(t)=$ $a\left(t+\tau_{L}\right)$, and $b(t)=b\left(t+\tau_{L}\right)$. The final stage of forming the initial quasiequilibrium is to load the particles with a Gaussian distribution in $\dot{x}$ and $\dot{y}$ and a uniform (stepfunction) density profile, into the matched envelope. Although the initial distribution described above does not provide an exact beam equilibrium, it still can be considered to provide a good approximation for a matched beam for present purposes. Moreover, to get rid of the possible effects induced by any initial mismatch, the beam is allowed to relax during 100 periods of the focusing lattice before compressing the lattice amplitude. The time dependence of the rms beam radius and the transverse emittance during the adiabatic and nonadiabatic processes including the first 100 lattice periods are shown in Fig. 6. For a nonaxysimmetric beam, which is studied in this section, we define the average beam radius as $R_{b}=\left(\tilde{a}^{2}+\tilde{b}^{2}\right)^{1 / 2}$, where $\tilde{a}=\left\langle x^{2}\right\rangle^{1 / 2}$ and $\tilde{b}=\left\langle y^{2}\right\rangle^{1 / 2}$ are the rms envelope dimensions. The average transverse emittance $\varepsilon$ is defined as $\varepsilon=\left(\varepsilon_{x} \varepsilon_{y}\right)^{1 / 2}$, where $\varepsilon_{x}=\left(\left\langle x^{2}\right\rangle\left\langle\dot{x}^{2}\right\rangle-\langle x \dot{x}\rangle^{2}\right)^{1 / 2}$ and $\varepsilon_{y}=\left(\left\langle y^{2}\right\rangle\left\langle\dot{y}^{2}\right\rangle-\langle y \dot{y}\rangle^{2}\right)^{1 / 2}$.

To model the lattice we take $k_{q}(t)=$ $2^{3 / 2} \pi \omega_{q} \tau_{L}^{-1} \sin \left(2 \pi t / \tau_{L}\right)$. All other parameters are the same as in Sec. II. It should be noted that even for nonadiabatic compression the transition time is sufficiently large so that the smooth-focusing approximation is valid during the transition phase. Comparing Figs. 4 and 6, we note that the smooth-focusing approximation and the full alternating-gradient quadrupole field model give remarkably similar results. The differences are evident in the emittance behavior during the initial stage (before beam 

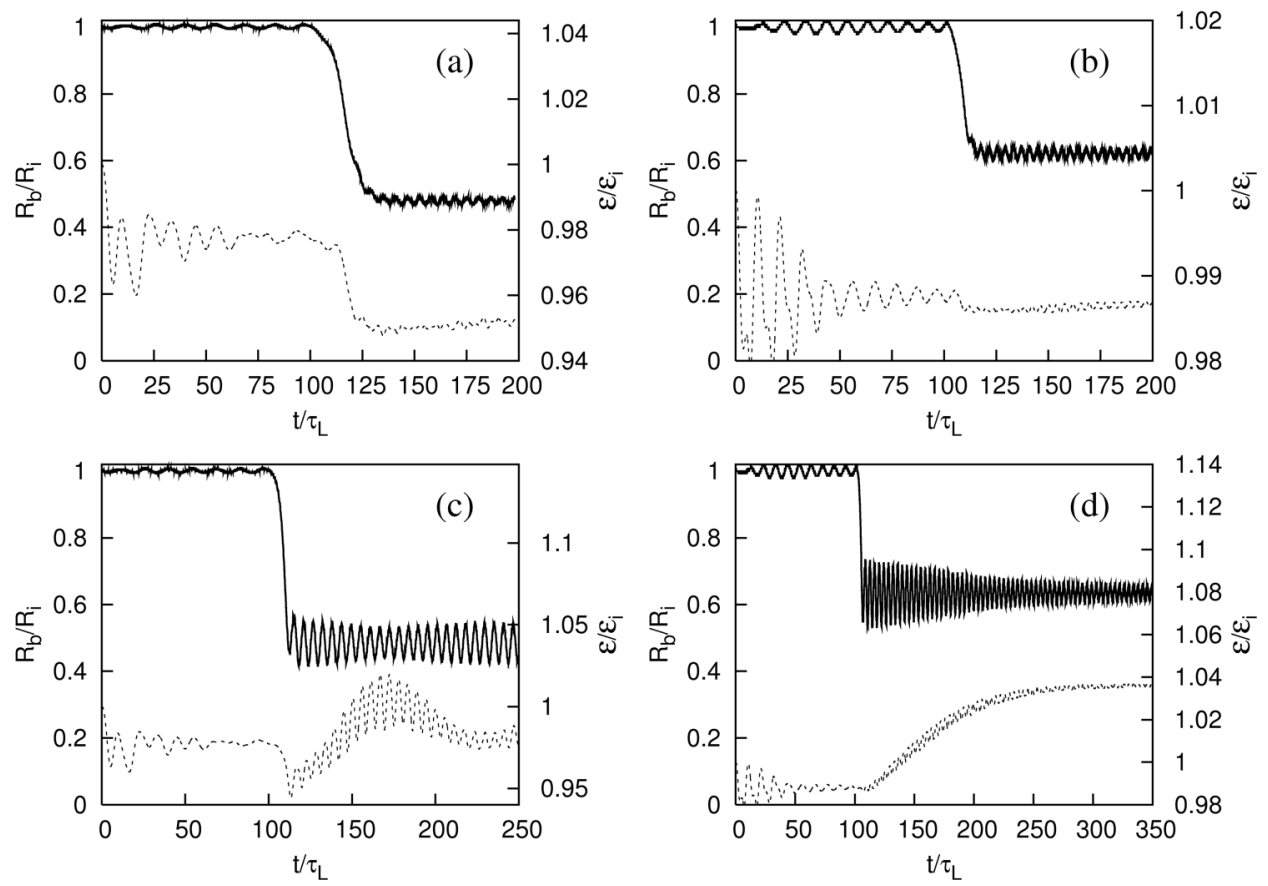

FIG. 6. Evolution of the normalized beam radius $R_{b} / R_{i}$ (solid line) and normalized transverse emittance $\varepsilon / \varepsilon_{i}$ (broken line) during the compression process. (a),(b) Correspond to an adiabatic compression for $s=0.9999, \tau_{1 / 2} / \tau_{L}=20$ [corresponding to $\tau_{q} \bar{\omega}\left(R_{0 i}\right)=$ 1.74], and $s=0.7, \tau_{1 / 2} / \tau_{L}=10$ [corresponding to $\tau_{q} \bar{\omega}\left(R_{0 i}\right)=1.28$ ], respectively. (c),(d) Correspond to a nonadiabatic compression for $s=0.9999, \tau_{1 / 2} / \tau_{L}=10$ [corresponding to $\tau_{q} \bar{\omega}\left(R_{0 i}\right)=0.87$ ], and $s=0.7, \tau_{1 / 2} / \tau_{L}=5$ [corresponding to $\tau_{q} \bar{\omega}\left(R_{0 i}\right)=0.64$ ], respectively. Results are obtained using the WARP code for an alternating-gradient quadrupole lattice.

propagation through the lattice transition region) which is due to the initial beam mismatch in the quadrupole field model. Furthermore, the smooth-focusing model shows a complete mixing of the oscillations in beam radius for a beam with moderate space-charge strength, $s=0.7$, during nonadiabatic compression, whereas in the quadrupole field model the amplitude of the oscillations only decreases to $30 \%$ of its initial value. However, contrary to the smooth-focusing approximation, the oscillations in average beam radius cannot be considered as a measure of the final-state mismatch. In fact, even for a perfectly matched beam ( $\mathrm{KV}$ distribution), the sum of the rms envelope dimensions is nearly constant, $\tilde{a}(t)+\tilde{b}(t) \cong$ const, but $R_{b}=\left[\tilde{a}^{2}(t)+\tilde{b}^{2}(t)\right]^{1 / 2} \neq$ const. Therefore, to estimate the mismatch of the final state, it is important to analyze the behavior of the rms envelope dimensions $\tilde{a}$ and $\tilde{b}$, which are illustrated in Figs. 7 and 8. It is evident from Fig. 7(b), which shows the time dependence of $\tilde{a}$ and $\tilde{b}$ for the nonadiabatic compression of a beam with $s=0.7$, that the beam is only slightly mismatched in the final state. The particle phase advances, defined as $\sigma=\varepsilon \int_{t}^{t+\tau_{L}} d t / a^{2}(t)=\varepsilon \int_{t}^{t+\tau_{L}} d t / b^{2}(t)$, and $\sigma_{\text {vac }}=$ $\lim _{K_{b} \rightarrow 0} \varepsilon \int_{t}^{t+\tau_{L}} d t / a^{2}(t)$ [1], are shown for the initial and final stages of compression process in Figs. 7 and 8. In Ref. [1] it was shown that the smooth-focusing estimate of $\sigma_{\text {vac }}$ is the approximately valid provided $\sigma_{\text {vac }} \leq 72^{0}$. Note in Figs. 7 and 8 that the lattice still satisfies this condition by the end of the transition region.

As mentioned earlier, for the parameters used in the simulations, the smooth-focusing approximation is valid during the transition phase even for a nonadiabatic process. This means that the perturbations introduced to the beam in the transition region of the quadrupole lattice can be averaged and the averaged perturbation has azimuthal symmetry. Therefore, as shown in Figs. 7 and 8, only the breathing mode ( $x$ and $y$ envelope dimensions oscillate with zero relative phase shift) is excited during the compression process.

\section{DETAILED STUDIES OF THE BEAM DISTRIBUTION FUNCTION DURING THE COMPRESSION PROCESS: HALO FORMATION}

In previous sections, the evolution of low-order moments of the distribution function (such as the rms envelope dimensions and transverse emittance) was studied. The results show that even nonadiabatic compression, which leads to significant beam mismatch by the end of the transition stage, does not result in large emittance growth $(\Delta \varepsilon<6 \%)$. Nevertheless, it is well known that a beam mismatch produces halo particles that have much higher transverse energies than the core particles and may cause a deterioration in beam quality during the subsequent 

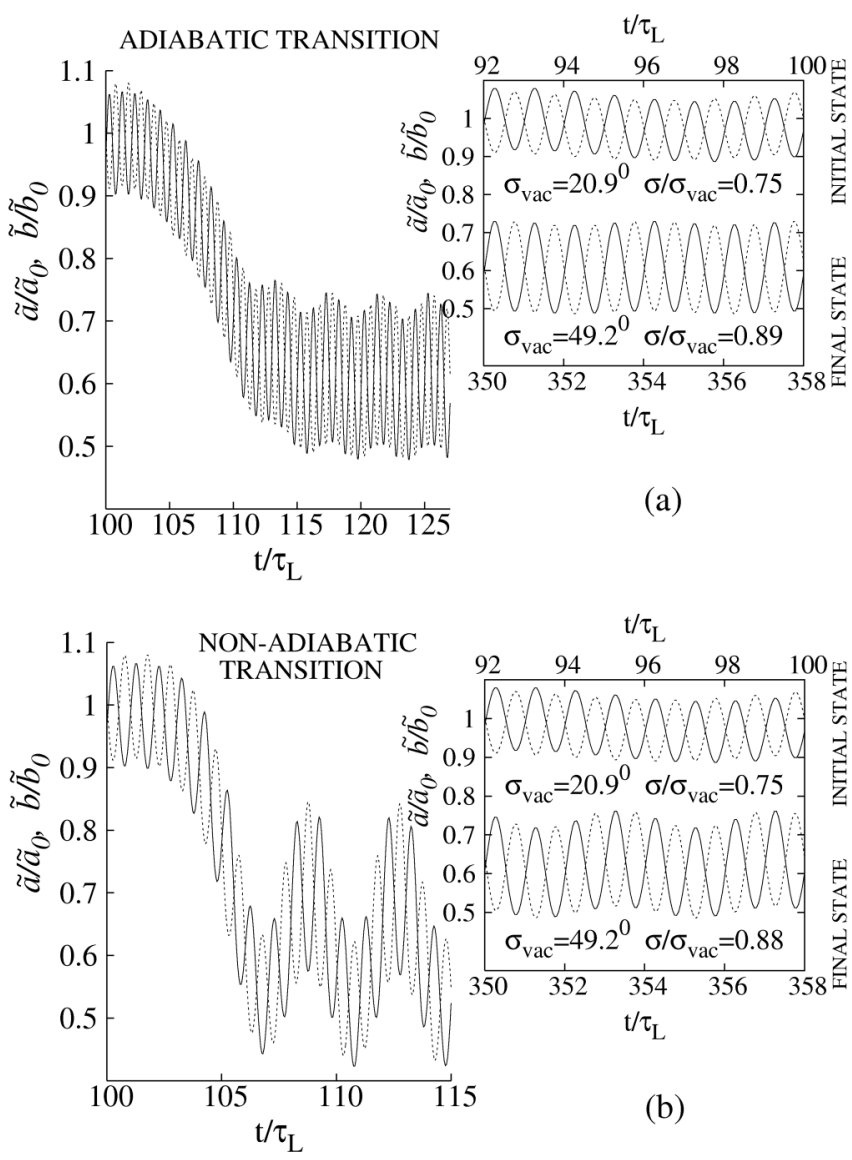

FIG. 7. WARP simulations of the beam compression in an alternating-gradient quadrupole lattice. Evolution of the rms envelope dimensions $\tilde{a} / \tilde{a}_{0}$ (solid line) and $\tilde{b} / \tilde{b}_{0}$ (broken line) are plotted during (a) adiabatic compression with $\tau_{1 / 2} / \tau_{L}=10$, and during (b) nonadiabatic compression with $\tau_{1 / 2} / \tau_{L}=5$, for a beam with moderate space-charge intensity, $s=0.7$.

beam transport. In this section, we present a detailed analysis of halo formation during the compression process using the WARP code for an alternating-gradient quadrupole lattice.

The main mechanism for halo formation, when a stable transport regime is maintained, is the resonance between the particle motion and a mismatch in the core oscillations. It is believed, for the case of a uniform density profile, that only particles that are outside the core and experience nonlinear forces can populate the halo. Other particles oscillate in the quadratic potential well and are trapped inside the core. During the compression process, due to the lack of adiabaticity and also due to nonuniformities in the density profile at the beam edge, some particles can leave the core and become highly energetic halo particles. Figures 9(a) and 9(b) and Figs. 10(a) and 10(b), respectively, illustrate the initial and final $(x, \dot{x})$ phase spaces for both moderate and high values of the space-charge intensity parameter $s$. The scaled coordinates $X=x /(2 \tilde{a})$ and $\dot{X}=2(\dot{x} \tilde{a}-x \dot{\tilde{a}}) / \varepsilon_{x}$ are plotted to remove the envelope oscillations.
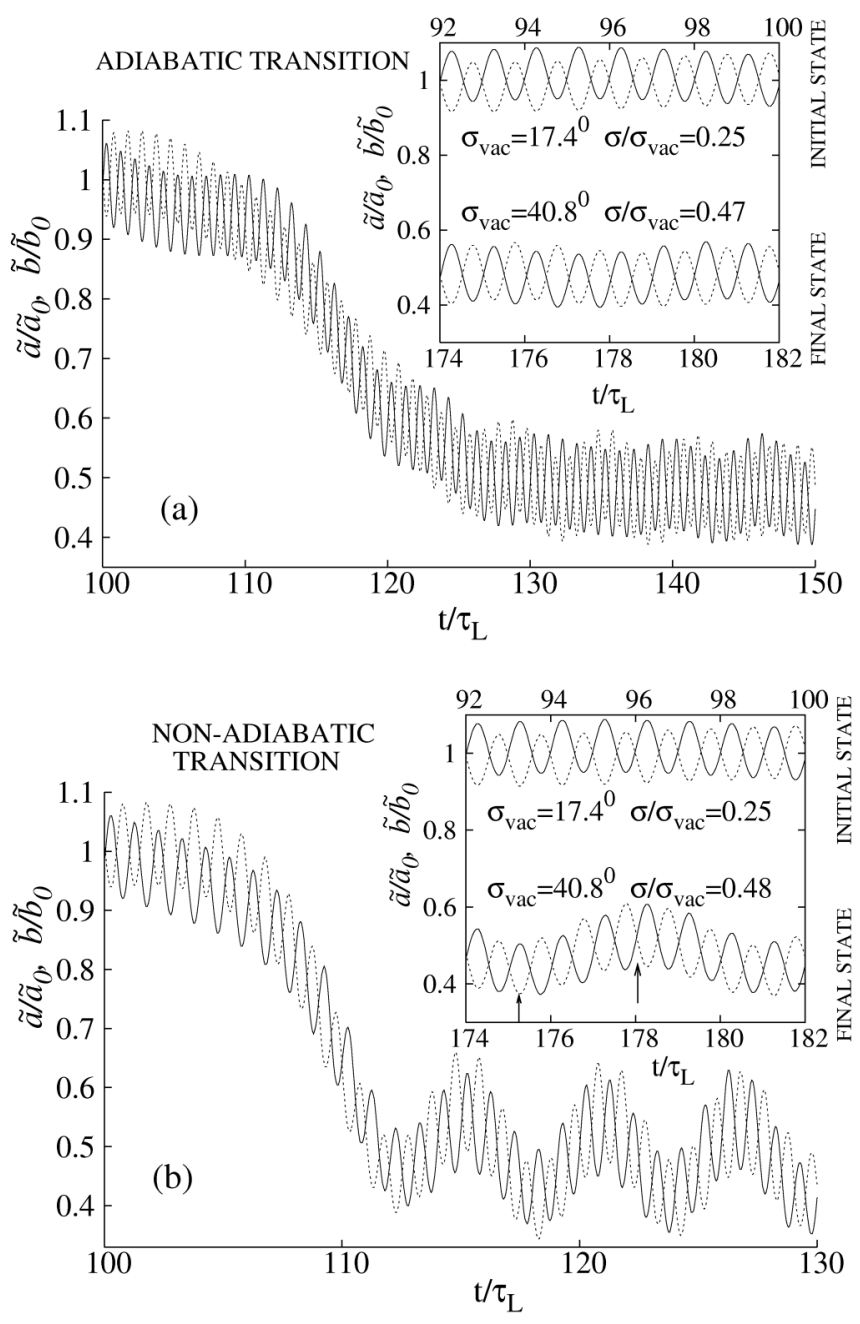

FIG. 8. WARP simulations of the beam compression in an alternating-gradient quadrupole lattice. Evolution of the rms envelope dimensions $\tilde{a} / \tilde{a}_{0}$ (solid line) and $\tilde{b} / \tilde{b}_{0}$ (broken line) are plotted during (a) adiabatic compression with $\tau_{1 / 2} / \tau_{L}=20$, and during (b) nonadiabatic compression with $\tau_{1 / 2} / \tau_{L}=10$, for a space-charge-dominated beam with $s=0.9999$.

For a space-charge-dominated beam with the almost flattop density profile shown in Fig. 11(a), the shape of the final phase-space plot [Fig. 10(b)] resembles a well-known Poincare section with a $2: 1$ resonance structure for the particle-core model with a KV core distribution. However, contrary to the particle-core model, where particles are usually launched with zero transverse canonical angular momentum, here the particle motion is in the 4D transverse phase space, providing a nearly complete smearing of the 2:1 resonance structure in the $X-\dot{X}$ phase-space projection. The halo evolution after one half-period of the mismatch oscillations is illustrated in Fig. 10(e). The time instants for the phase-space plots are indicated by arrows in Fig. 8(b) for the rms envelope dimensions in the final state.

Note that the 2:1 resonance points are located on the $X$ axis when the core radius is a minimum, and on the $\dot{X}$ axis when the core radius is a maximum. This coincides well 

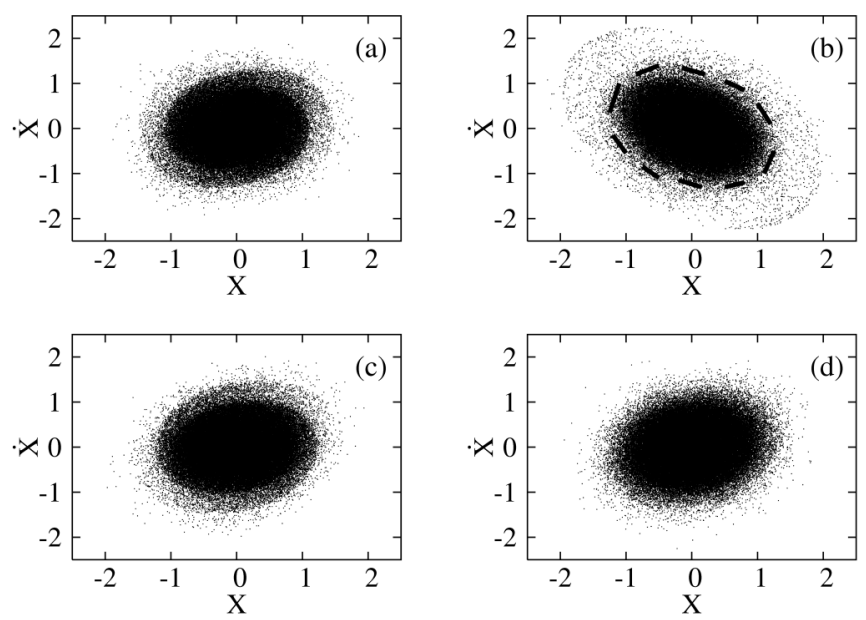

FIG. 9. Plots of the instantaneous $(X, \dot{X})$ phase space for a beam with moderate space-charge intensity, $s=0.7$ : (a) Initial state at $t / \tau_{L}=100$; (b) final state at $t / \tau_{L}=357.3$ for nonadiabatic compression with $\tau_{1 / 2} / \tau_{L}=5$; (c) final phase of the transition stage at $t / \tau_{L}=110$ for nonadiabatic compression with $\tau_{1 / 2} / \tau_{L}=5$; (d) final state at $t / \tau_{L}=309.95$ for adiabatic compression with $\tau_{1 / 2} / \tau_{L}=10$.

with the results obtained by Ikegami in [12], where the Poincare section for the particle-core model with the strobe time, taken at the minimum (maximum) of the beam size, gave the same location of the resonance islands. In the same work [12], the maximum halo extent (the width of the separatrix of the 2:1 resonance island) was found to be about twice as large as the maximal core radius, when the halo was driven by the breathing mode. Figure 10(b) illustrates approximately the same halo width. The analogous studies were also performed for a beam with moderate space-charge strength, $s=0.7$, and the results are illustrated in Fig. 9. The strong nonuniformities in the density profile [compare Figs. 11(a) and 11(b)] lead to a complete mixing of the mismatch oscillations, and therefore particles do not experience resonance interaction in the final state [Fig. 9(b)].

To assure that the simulation parameters do demonstrate halo formation, and that the above analysis is not a collateral effect due to the core tails, we use a smooth-focusing model with the same parameters and plot the radial $r-\dot{r}$ phase space, (see Fig. 12). Note that in the smoothfocusing approximation there is no core flutter and we do not use the scaled coordinates. Figure 12(a) for a spacecharge-dominated beam with $s=0.9999$ clearly illustrates the resonance structure, and the resonance structure is not observed in Fig. 12(b) for moderate space-charge intensity with $s=0.7$. In Sec. II B it was indicated that the relaxation of a large mismatch for a space-charge-dominated beam corresponds qualitatively to the nonlinear stage of Landau damping. Indeed, the halo particles illustrated in Fig. 12(a) are the trapped particles in the nonlinear interaction between the collective mismatch oscillations and the single particle motion.

We emphasize here some interesting features of the halo formation. The simulations show that during the transition stage only a small number of particles leave the core [compare Figs. 9(a) and 9(c) and Figs. 10(a) and 10(c)], whereas most particles populate the halo region during the
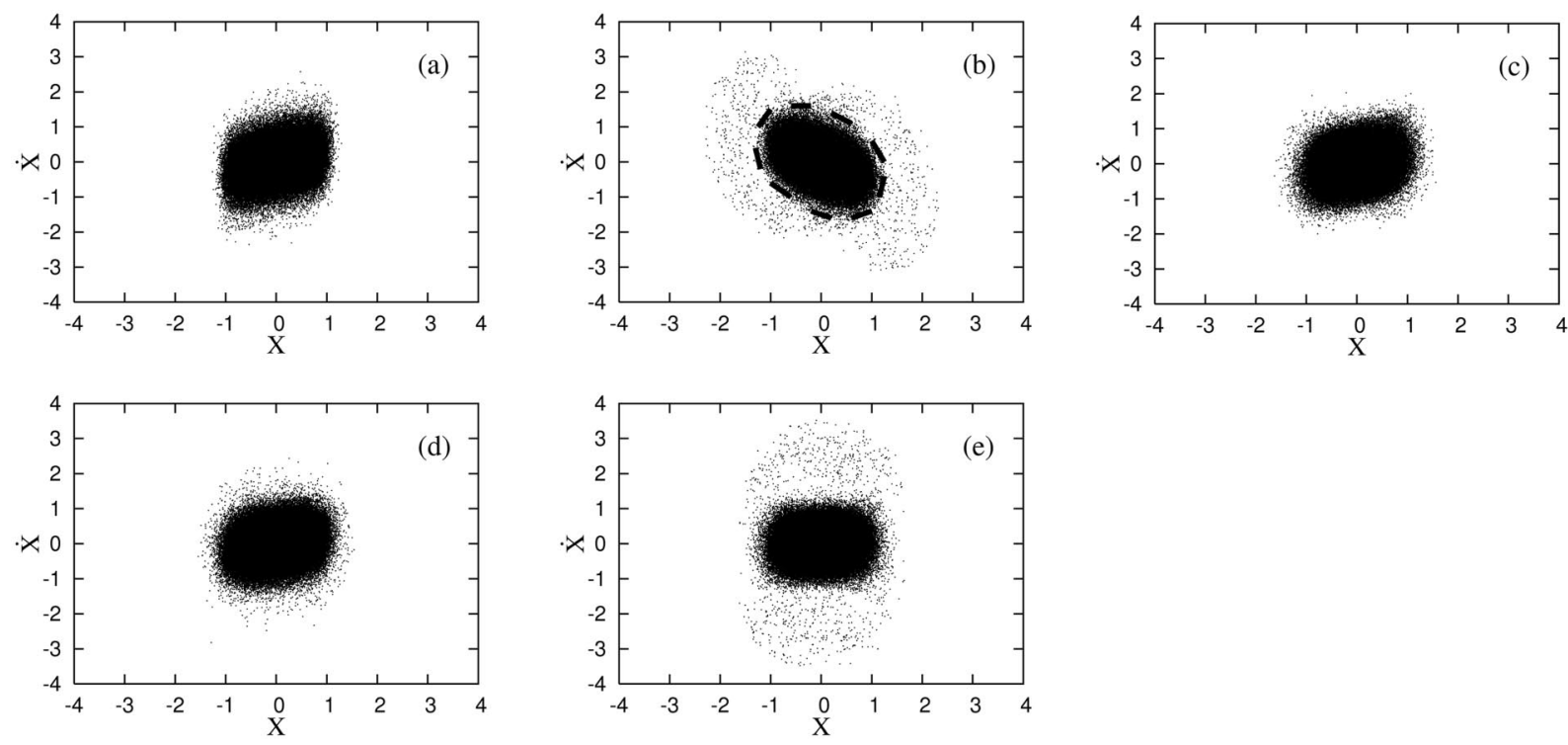

FIG. 10. Plots of the instantaneous $(X, \dot{X})$ phase space for a space-charge-dominated beam, $s=0.9999$ : (a) Initial state at $t / \tau_{L}=$ 100; (b) final state at $t / \tau_{L}=175.25$ (corresponds to the minimum beam radius) for nonadiabatic compression with $\tau_{1 / 2} / \tau_{L}=10$; (c) final phase of the transition stage at $t / \tau_{L}=120$ for nonadiabatic compression with $\tau_{1 / 2} / \tau_{L}=10$; (d) final state at $t / \tau_{L}=199$ for adiabatic compression with $\tau_{1 / 2} / \tau_{L}=20$; (e) final state at $t / \tau_{L}=178.05$ (corresponds to the maximum beam radius) for nonadiabatic compression with $\tau_{1 / 2} / \tau_{L}=10$. 

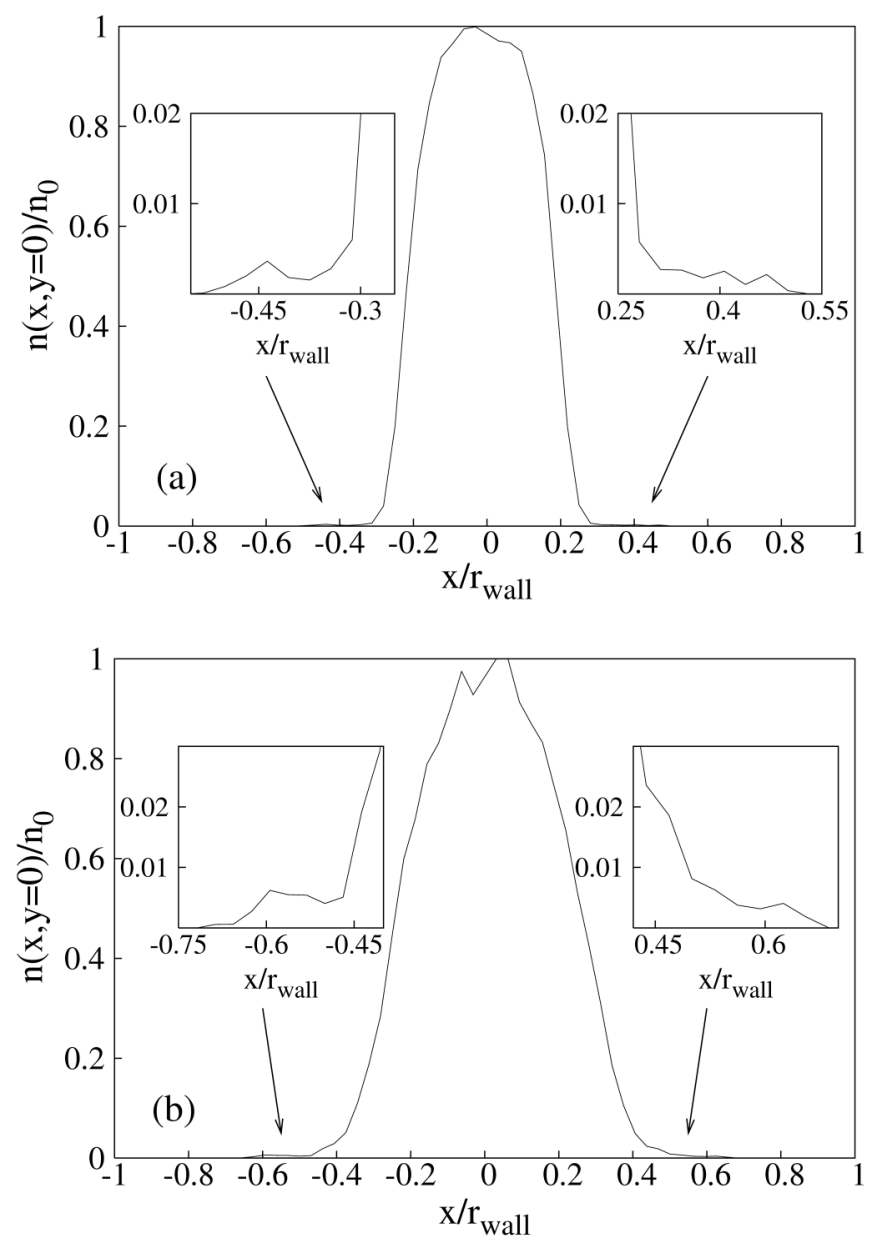

FIG. 11. WARP simulation for an alternating-gradient quadrupole lattice. Normalized beam density profile $n(x, y=0) / n_{0}$ in the final state for nonadiabatic compression. The two cases correspond to (a) $s=0.9999, \tau / \tau_{L}=175.25$, and (b) $s=0.7$, $\tau / \tau_{L}=375.3$. The small graphical inserts correspond to the density profile at the core edge.

relaxation stage [compare Figs. 9(a) and 9(b) and Figs. 10(a) and 10(b)]. Furthermore, it is found that the halo formation process saturates along with the Landau damping of the mismatch mode. These details are evidence that the main mechanism for halo formation is indeed an energy transfer from the collective modes to the resonant particles (halo particles). When the transition stage is much shorter than the characteristic Landau damping time, there is negligible collective energy transfer to the particles, and the particles stay trapped inside the beam core.

Of considerable practical interest are the halo particle contributions to the low-order moments of the beam distribution function. To investigate these phenomena qualitatively, we have also arbitrary removed the halo particles from the simulation. No rigorous mathematical criteria were applied for the removal procedure. We simply removed particles from the $X-\dot{X}$ and $Y-\dot{Y}$ projections of the $4 \mathrm{D}$ phase space using "visual criteria" to obtain approxi-
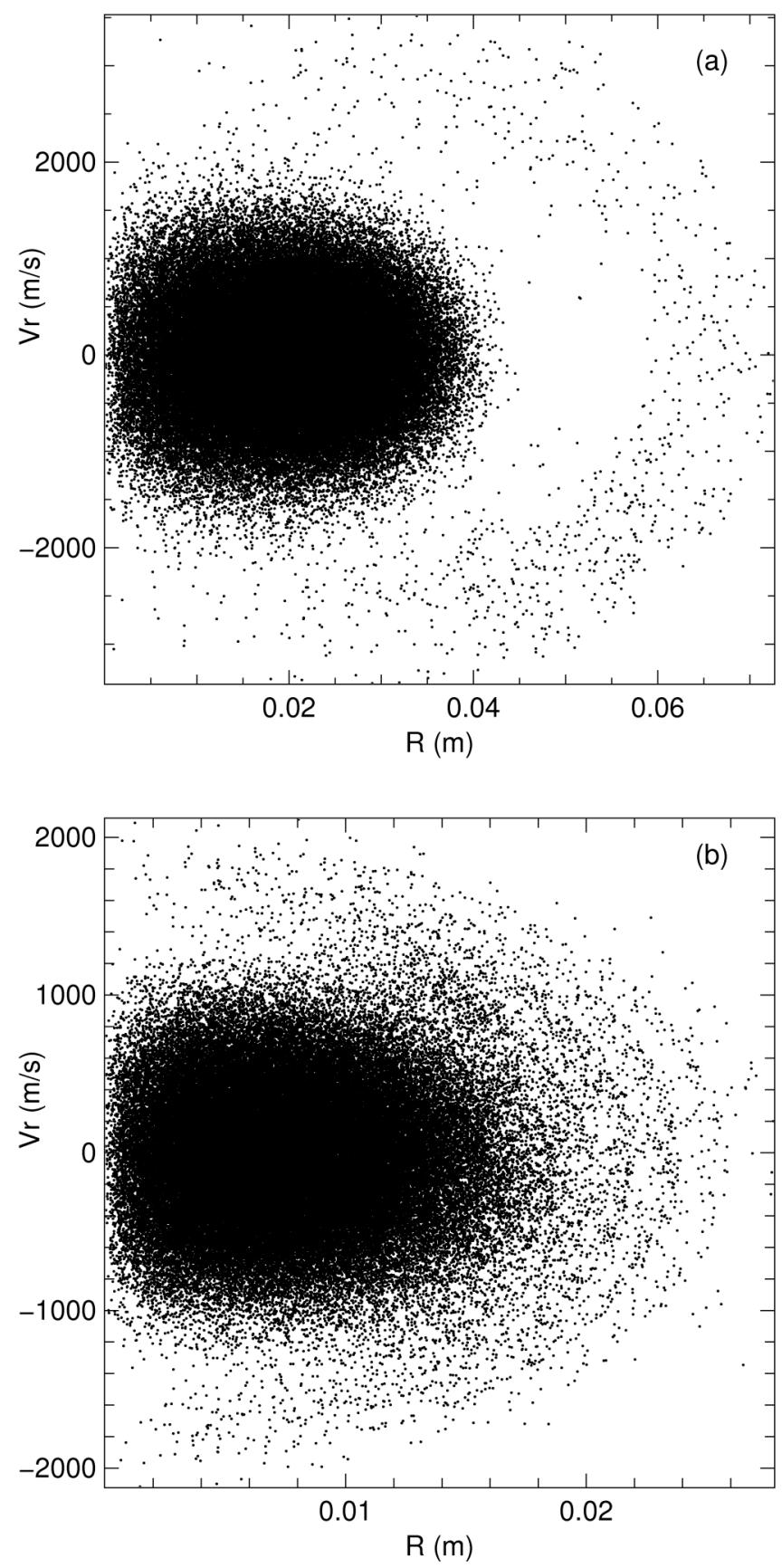

FIG. 12. WARP simulation results using a smooth-focusing model. Plots of the radial $(r, \dot{r})$ phase space at the final state of the nonadiabatic compression process for (a) $s=0.9999$, $t / \tau_{L}=437.5$, and (b) $s=0.7, t / \tau_{L}=725.5$.

mate quantitative information about interesting phenomena. The contours which were used to divide the $X-\dot{X}$ phase space into the core and halo regions are illustrated in Figs. 9(b) and 10(b), and analogous contours were applied to the $Y-\dot{Y}$ phase-space projection. The ratios of the values of rms envelope dimensions calculated with and without halo particles are $\tilde{a}^{\text {without halo }} / \tilde{a}^{\text {with halo }}=0.95$ for $s=0.9999$, and $\tilde{a}^{\text {without halo }} / \tilde{a}^{\text {with halo }}=0.91$ for $s=0.7$. 
For the transverse emittance we obtained $\varepsilon_{x}^{\text {without halo }} / \varepsilon_{x}^{\text {with halo }}=0.9 \quad$ for $\quad s=0.9999, \quad$ and $\varepsilon_{x}^{\text {without halo }} / \varepsilon_{x}^{\text {with halo }}=0.85$ for $s=0.7$. It should be emphasized here that, after halo removal, the emittance drops somewhat below its initial value. Consequently, for the parameters used here, the core size does not grow in phase space during the relaxation stage, but the halo formation provides an increase in the total beam phase-space area and causes the growth of the emittance. The halo fraction of all simulation particles is about $2 \%$ for a space-charge-dominated beam with $s=0.9999$, and about $4 \%$ for a moderate intensity beam with $s=0.7$. This difference can be explained by recalling (Sec. II B) that more energy transfers from the collective oscillations to the transverse particle motion for $s=0.7$ than for $s=0.9999$. Another interesting feature is that a negligible number of new halo particles (less than $0.05 \%$ of all simulation particles) are observed for both values of $s$ if we continue the simulations after the halo particle removal procedure (in these simulations, to conserve the line-charge, we placed the removed halo particles on the beam axis). This indicates that, despite charge density nonuniformities at the beam edge and mismatch oscillations, the phase space of the beam core is surrounded by a KAM surface, providing the core particles stay inside the core region and do not penetrate the halo region.

The results for adiabatic compression for different values of the space-charge intensity parameter are illustrated in Figs. 7(a) and 8(a) (rms envelope dimensions behavior) and Figs. 9(d) and 10(d) $(X-\dot{X}$ phase space). These figures show that a certain level of the final beam mismatch still persists. However, it should be noted that the final population of halo particles is similar to the initial one, which is due to the challenge in loading the initial "equilibrium." Hence, a further increase in the transition time does not lead to an improved quality of the final beam state.

\section{CONCLUSIONS}

Envelope equations and full particle-in-cell numerical simulations using the WARP code have been used to investigate the evolution of the rms beam radius, the emittance growth, and halo formation during the transverse compression of an intense ion beam propagating through an alternating-gradient quadrupole lattice. It was shown that when the lattice transition is smooth (adiabatic) the emittance variation is negligibly small, and therefore a constant-emittance approximation can be used as a closure condition for the envelope equations to model the compression process. For the case of a nonadiabatic transition, it was found that the characteristic time scale for the emittance growth is much larger than the transition time required for adiabatic compression. Therefore, even for nonadiabatic compression, the constant-emittance approximation can be used to estimate the beam mismatch produced in the transition region.
The details of halo formation were investigated selfconsistently using the WARP code, both in the smoothfocusing approximation and for a quadrupole lattice. In the smooth-focusing approximation, a 2:1 resonance structure was observed for space-charge-dominated beams with almost uniform density profile. For a quadrupole lattice, the beam particle motion in the $4 \mathrm{D}$ transverse phase space provides some smearing of the 2:1 resonance structure in the $2 \mathrm{D}$ phase-space projection. Nonetheless the width and location of the resonance islands coincide well with the results, obtained by Ikegami [12] in the particlecore model for a quadrupole focusing field. It was also found that during halo formation the energy transfers from the collective mismatch oscillations to the transverse motion of the resonant particles (halo particles). The energy transfer time is of order the phase-mixing (Landau damping) time. Therefore, only a few particles populate the halo region during beam propagation through the lattice transition region. Generation of most of the halo particles, and consequently growth of the transverse emittance, occurs during the subsequent beam transport.

It was also found in the smooth-focusing approximation (constant focusing frequency) that the collective relaxation of the mismatch oscillations of a space-charge-dominated beam saturates with the formation of a stable, transverse, nonlinear wave structure.

\section{ACKNOWLEDGMENTS}

The authors are grateful to Dave Grote for his expert services in implementation of the WARP code. This research was supported by the U.S. Department of Energy.

[1] R. C. Davidson and H. Qin, Physics of Intense Charged Particle Beams in High Energy Accelerators (World Scientific, Singapore, 2001), and references therein.

[2] M. Reiser, Theory and Design of Charged Particle Beams (John Wiley \& Sons, Inc., New York, 1994).

[3] A. W. Chao, Physics of Collective Beam Instabilities in High Energy Accelerators (John Wiley \& Sons, Inc., New York, 1993).

[4] E. Henestroza, S. Eylon, P. K. Roy, S. S. Yu, A. Anders, F. M. Bieniosek, W. G. Greenway, B. G. Logan, R. A. MacGill, D. B. Shuman, D. L. Vanecek, W. L. Waldron, W. M. Sharp, T.L. Houck, R.C. Davidson, P.C. Efthimion, E. P. Gilson, A. B. Sefkow, D. R. Welch, D. V. Rose, and C.L. Olson, Phys. Rev. ST Accel. Beams 7, 083501 (2004).

[5] P. K. Roy, S. S. Yu, S. Eylon, E. Henestroza, A. Anders, F. M. Bieniosek, W. G. Greenway, B. G. Logan, W. L.Waldron, D. L. Vanecek, D. R. Welch, D. V. Rose, R.C. Davidson, P.C. Efthimion, E.P. Gilson, A.B. Sefkow, and W. M. Sharp, Phys. Plasmas 11, 2890 (2004).

[6] I. Kaganovich, E. A. Startsev, R. C. Davidson, and D. R. Welch, Nucl. Instrum. Methods Phys. Res., Sect. A 544, 255 (2005). 
[7] Hong Qin, Ronald C. Davidson, John J. Barnard, and Edward P. Lee, Phys. Rev. ST Accel. Beams 7, 104201 (2004).

[8] Robert L. Gluckstern, Phys. Rev. Lett. 73, 1247 (1994).

[9] T.P. Wangler, K. R. Crandall, R. Ryne, and T.S. Wang, Phys. Rev. ST Accel. Beams 1, 084201 (1998).

[10] J. Qiang, P. L. Colestock, D. Gilpatrick, H. V. Smith, T. P. Wangler, and M.E. Schulze, Phys. Rev. ST Accel. Beams 5, 124201 (2002).

[11] D. P. Grote, A. Friedman, I. Haber, W. Fawley, and J.-L. Vay, Nucl. Instrum. Methods Phys. Res., Sect. A 415, 428 (1998).

[12] Masanori Ikegami, Phys. Rev. E 59, 2330 (1999).

[13] M. G. Tiefenback and D. Keefe, IEEE Trans. Nucl. Sci. 32, 2483 (1985).
[14] M.G. Tiefenback, Ph.D. thesis, UC Berkeley, 1986; Lawrence Berkeley Lab Report No. LBL-22465, 1986.

[15] S. M. Lund and B. Bukh, Phys. Rev. ST Accel. Beams 7, 024801 (2004).

[16] E. P. Gilson, R.C. Davidson, P.C. Efthimion, and R. Majeski, Phys. Rev. Lett. 92, 155002 (2004).

[17] E. P. Gilson, M. Chung, R. C. Davidson, P. C. Efthimion, R. Majeski and E. A. Startsev, Nucl. Instrum. Methods Phys. Res., Sect. A 544, 171 (2005).

[18] T. Clauser, V. Stagno, and V. Variale, in Proceedings of the Particle Accelerator Conference, New York, 1999 (IEEE, Piscataway, NJ, 1999), p. 1779.

[19] V. Variale, Phys. Rev. ST Accel. Beams 4, 084201 (2001). 